\title{
Uso dos anti-inflamatórios não esteroides no controle da dor aguda: revisão sistemática
}

\author{
Use of nonsteroidal anti-inflammatory drugs to control acute pain: a systematic review \\ Washington Luis Melo Figueiredo ${ }^{1}$, Túlio César Azevedo Alves ${ }^{2}$
}

\section{RESUMO}

Objetivo. Revisar na literatura a eficácia e a segurança dos AINEs no controle da dor aguda, quando usados isoladamente ou através da analgesia multimodal. Método. Revisão sistemática sobre o uso dos anti-inflamatórios não esteroides (AINEs) no controle da dor aguda, usando as seguintes bases de dados e ferramentas de pesquisa: Medline, Scientific Eletronic Library Online (Scielo), Lilacs e Pubmed. A pesquisa foi realizada de outubro de 2014 a março de 2015 nos idiomas português e/ou inglês, publicados há no máximo 5 anos. Resultados. Foram analisados 16 artigos, entre os quais 12 (68,75\%), abordaram AINEs classificados como "eficaz" no controle da dor aguda, quando usados de forma isolada ou de forma combinada a outros fármacos, $0(0 \%)$ como "ineficaz", 1 (6,25\%) como eficácia "moderada" e 4 (25\%) trabalhos classificados como eficácia "não avaliada", quanto a segurança, 9 (56,25\%) artigos foram classificados como "seguro", $3(18,75 \%)$ como "inseguro", 1 (6,25\%) como "não estabelecida" a segurança e $3(18,75 \%)$ como segurança "não avaliada". Conclusáo. Notou-se uma maior prevalência quanto à eficácia e a segurança dos anti-inflamatórios não esteroides no controle da dor aguda de leve a moderada intensidade, tanto através do seu uso isolado, quanto através da sua combinação multimodal.

Unitermos. Anti-inflamatórios Não esteroides, Dor Aguda, Tratamento Multimodal, Efeitos Adversos

Citaçáo. Figueiredo WLM, Alves TCA. Uso dos anti-inflamatórios não esteroides no controle da dor aguda: Revisão sistemática.

Trabalho realizado na Universidade Estadual do Sudoeste da Bahia, Bahia-BA, Brasil.

1. Discente do curso de Medicina, Departamento de Saúde, Universidades Estadual do Sudoeste da Bahia, Uesb, Salvador-BA, Brasil.

2. Doutor, Professor da disciplina de Anestesiologia do Departamento de Saúde do Universidade Estadual do Sudoeste da Bahia - UESB, Salvador-BA, Brasil.

\begin{abstract}
Objective. Reviewing in the literature the efficacy and safety of Nonsteroidal Anti-inflammatory Drugs (NSAIDs) in acute pain control when used alone or by multimodal analgesic. Method. Systematic review of the use of nonsteroidal anti-inflammatory drugs (NSAIDs) to control acute pain, using the following databases and research tools: Medline, Scientific Eletronic Library Online (Scielo), Lilacs, and Pubmed. The survey was conducted from October 2014 to March 2015 in Portuguese and/or English, published at most five years ago. Results. We analyzed 16 articles, of which 12 (68.75\%), addressed NSAIDs classified as "effective" to control acute pain, when used alone or in combination with other drugs, $0(0 \%)$ as "ineffective" 1 $(6.25 \%)$ as effective "moderate" and $4(25 \%)$ classified as work effectiveness "not evaluated" for safety, 9 (56.25\%) articles were classified as "safe", 3 (18.75\%) as "unsafe", 1 (6.25\%) as "not established" security and $3(18.75 \%)$ as security "not evaluated". Conclusion. It was noted a higher prevalence for efficacy and safety of nonsteroidal anti-inflammatory drugs to control acute pain of mild to moderate intensity, both through its use alone, as through its multimodal combination.
\end{abstract}

Keywords. Anti-inflammatory Drugs, Acute Pain, Multimodal Treatment, Side Effects

Citation. Figueiredo WLM, Alves TCA. Use of nonsteroidal anti-inflammatory drugs to control acute pain: a systematic review. Endereço para correspondência:
Túlio César Azevedo Alves
Horto Florestal
R. Conselheiro Correia de Menes, 66
CEP 40295-030, Salvador-BA
Tel.: (71) 9115-2407
Email: tulio.alves@terra.com.br
Revisão
Recebido em: 11/05/15
Aceito em: 18/08/15
Conflito de interesses: não 
INTRODUÇÃO

Os anti-inflamatórios náo esteroides (AINEs) estão entre os fármacos mais amplamente usados no mundo ${ }^{1}$. Eles possuem propriedades anti-inflamatórias, analgésicas e antitérmicas ${ }^{2,3}$, tornando-se, úteis, portanto, para o controle da dor aguda. Com frequência, são prescritos para aliviar queixas musculoesqueléticas e são amiúde tomados sem prescrição médica para dores de menor intensidade ${ }^{2}$. Tais fármacos representam um grupo de compostos quimicamente heterogêneos, que frequentemente não têm relação química entre si, mas compartilham certas açóes terapêuticas e determinados efeitos adversos que estão relacionados principalmente com o uso indiscriminado ou períodos prolongados. Todos esses fármacos são inibidores da enzima ciclooxigenase (COX) e podem produzir reações adversas como gastrite, úlceras gástricas, perfuração gastrointestinal, disfunção plaquetária, hemorragia e comprometimento renal ${ }^{1-3}$.

De maneira geral, os AINES, incluindo a subclasse de inibidores seletivos da ciclooxigenase 2 (COX2), são amplamente utilizados no controle da dor aguda. A dor aguda resulta da ativação dos aferentes nociceptivos periféricos através de estímulos nocivos (potencialmente perigosos) assumindo a sensibilidade na linha de base do sistema sensorial e ajuda a prevenir o dano tecidual iminente. Adicionalmente, a dor aguda pode em certas circunstâncias evoluir para a dor crônica ${ }^{4,5}$.

Para o alívio da dor aguda, os AINEs podem ser utilizados isoladamente, associados a outros analgésicos ou em combinação com a analgesia regional. Possuem, ainda, as vantagens de não causar dependência, depressáo respiratória, além de reduzir a dose necessária de analgésicos opioides, quando associados a estes, resultando assim, em menos efeitos adversos. Na estratégia do controle multimodal da dor, os AINES têm sido utilizados em associação com outros fármacos resultando em efeito sinérgico com melhor controle da dor pós-operatória e diminuição dos efeitos adversos ${ }^{6,7}$.

Por outro lado, o uso isolado dos AINEs, em grandes doses ou em pacientes susceptíveis, pode produzir importantes reaçóes adversas. Desse modo, o uso indiscriminado desses fármacos pode comprometer a relação custo-benefício, tornando-se prejudicial para o paciente $^{8,9}$.
Portanto, este artigo tem como objetivo revisar a literatura acerca da eficácia e segurança dos AINEs no controle da dor aguda, quando usados isoladamente ou através da analgesia multimodal.

\section{MÉTODO}

Trata-se de uma revisão sistemática realizada em base de dados aberta, através da busca de referências sobre o uso dos anti-inflamatórios não esteroides (AINEs) no controle da dor aguda, usando as seguintes bases de dados e ferramentas de pesquisa: Medline, Scientific Eletronic Library Online (Scielo), Lilacs e Pubmed. Os descritores usados foram: anti-inflamatórios não esteroides - dor aguda analgesia multimodal - efeitos colaterais dos AINEs - dor aguda e analgesia multimodal - dor aguda OR analgesia multimodal - dor aguda AND anti-inflamatórios não esteroides - dor aguda OR anti-inflamatórios não esteroides - anti-inflamatórios não esteroides AND efeitos colaterais dos AINEs - anti-inflamatórios não esteroides OR efeitos colaterais dos AINEs - dor aguda AND NOT analgesia multimodal.

A pesquisa foi realizada de outubro de 2014 a março de 2015, sendo selecionados artigos de revisão sistemática, estudos comparativos, ensaios clínicos controlados e/ou estudos de coorte. Para tanto, utilizou-se os idiomas português e/ou inglês, publicados há no máximo 5 anos (2009 a 2014).

Para a coleta dos materiais foi feita a leitura do título e do resumo, sendo os critérios de inclusão: estudos que associaram AINEs com dor aguda, AINEs e analgesia multimodal, AINEs com efeitos colaterais, dor aguda com analgesia multimodal, estudos em humanos adultos e textos completos disponíveis. Os critérios de exclusão foram: estudos com animais, estudos com crianças, relatos de caso e artigos incompletos. A análise dos dados foi realizada através da análise de conteúdo de Bardin.

Para a análise dos artigos foram criadas quatro categorias referentes à eficácia dos AINEs: 1. eficaz, artigos que abordaram AINEs capazes de aliviar a dor aguda; 2. ineficaz, artigos que abordaram AINEs que não foram capazes de aliviar a dor aguda; 3. moderada, artigos que abordaram AINEs que aliviaram a dor aguda momentaneamente, mas a mesma apresentou elevada taxa de retor- 
no e 4. não avaliada, artigos que não abordaram a eficácia dos AINEs, mas avaliaram a segurança dos mesmos. Do mesmo modo, também foram criadas quatro categorias para segurança: 1. seguro, artigos que abordaram AINEs sem efeitos colaterais graves; 2. inseguro, artigos que abordaram AINEs com efeitos colaterais graves; 3. não estabelecida, artigos que apontaram a necessidade de novos estudos quanto a segurança e 4. não avaliada, artigos que não avaliaram a segurança dos AINEs.

\section{RESULTADOS}

$\mathrm{Na}$ Scielo, foram encontrados 163 artigos, selecionados 5, após filtragem e excluídos 158. Na Lilacs, de 106 estudos, 3 foram selecionados e 103 excluídos. Na PubMed, 1363 encontrados, sendo 4 incluídos e 1359 excluídos. Na Medline, localizou-se 434, selecionou-se 6 e excluiu-se 428. Nesta fase, foram excluídos 2 artigos repetidos.

Nesta revisão foram analisados 16 artigos, entre os quais 12 (68,75\%), abordaram AINEs classificados como "eficaz" no controle da dor aguda, quando usados de forma isolada ou de forma combinada a outros fármacos, 0 (0\%) como "ineficaz", 1 (6,25\%) como eficácia "moderada” e 4 (25\%) trabalhos classificados como eficácia "não avaliada", conforme Quadro 1.

Outro aspecto abordado foi a segurança dos AINEs, deste modo, $9(56,25 \%)$ artigos foram classificados como "seguro", 3 (18,75\%) como "inseguro", 1 (6,25\%) como "não estabelecida" a segurança e 3 (18,75\%) como segurança "não avaliada".

\section{DISCUSSÃO}

Os AINEs mostram-se principalmente eficazes no controle da dor associada à inflamação ou à lesão tecidual, visto que diminuem a produção das prostaglandinas que sensibilizam os nociceptores a mediadores da inflamação, como a bradicinina. Por outro lado, são responsáveis por numerosas reaçôes adversas, principalmente quando são utilizados por longos períodos ou na presença de fatores de risco para estas reaçóes ${ }^{1-3}$.

Com relação ao efeito analgésico, os resultados mostram que $68,75 \%$ dos artigos estudados evidencia-
Quadro 1. Eficácia e segurança dos AINEs conforme o autor e ano.

\begin{tabular}{|c|c|c|}
\hline Autor & Eficácia & Segurança \\
\hline Bidese et al, $2014^{10}$ & Eficaz & Seguro \\
\hline Desai et al, $2014^{11}$ & Eficaz & Seguro \\
\hline Barbosa et al, $2014^{12}$ & Moderada & Seguro \\
\hline Sertoz et al, $2013^{13}$ & Eficaz & Seguro \\
\hline Kara et al, $2010^{14}$ & Eficaz & Seguro \\
\hline Husted et al, $2011^{15}$ & Eficaz & Seguro \\
\hline Defrance et al, $2011^{16}$ & Não avaliada & Seguro \\
\hline Lee et a et al, $2013^{17}$ & Eficaz & Seguro \\
\hline Lee et al, $2011^{18}$ & Não avaliada & Inseguro \\
\hline $\begin{array}{c}\text { Nezvalová-Henriksen } \\
\text { et al, } 2013^{19}\end{array}$ & Eficaz & Não estabelecida \\
\hline Oslen et al, $2012^{20}$ & Não avaliada & Inseguro \\
\hline Andersen et al, $2011^{21}$ & Eficaz & Não avaliada \\
\hline Mezentseve, $2009^{22}$ & Eficaz & Seguro \\
\hline Clan et al, $2011^{23}$ & Não avaliada & Inseguro \\
\hline Vega et al, $2013^{24}$ & Eficaz & Não avaliada \\
\hline Boonriong et al, $2010^{25}$ & Eficaz & Não avaliada \\
\hline
\end{tabular}

ram a eficácia dos anti-inflamatórios não esteroides no controle da dor aguda. Adicionalmente, verificou-se efeito sinérgico entre AINEs e opioide ${ }^{10}$, com redução da prevalência da dor pós-operatória de 59\%, quando era utilizado o AINE isoladamente, para 22\%, quando o opioide era associado ao AINE. Observou-se, ainda, um efeito sinérgico entre AINE e anestésico local ${ }^{11}$, porém limitado às seis primeiras horas de pós-operatório.

Adicionalmente, nenhum artigo foi classificado como ineficaz. Sendo assim, todos os fármacos administrados aliviaram a dor em proporçóes diferentes, ainda que por tempo determinado. Nesse contexto, 6,25\% dos artigos encontraram eficácia moderada, a qual foi atribuída à alta prevalência de dor no período pós-operatório de cirurgias ortopédicas. A dor estava presente em 65,7\% dos pacientes no pós-operatório imediato, $62,2 \%$ no primeiro dia de pós-operatório e de $75 \%$ no segundo dia de pós-operatório ${ }^{12}$.

No aspecto segurança, verificou-se que os AINEs foram considerados seguros em 56,25\% dos artigos selecionados, isoladamente ou em associação com os analgésicos opioides. Quando associados aos opioides, alguns efeitos adversos foram encontrados em maior proporção, como prurido, retenção urinária, náusea e, pelo menos um episódio de vômito ${ }^{10-15}$.

Observou-se, ainda, em menor proporção, rea- 
çóes cutâneas como a urticária e exantema, atribuídas, sobretudo, ao uso de ácido acetilsalicílico, paracetamol, ibuprofeno e piroxicam ${ }^{16}$. Estas reaçóes não foram consideradas graves.

Em outro grupo de pacientes, verificou-se náuseas, vômitos, constipação, retenção urinária e sonolência quando os AINEs foram associados à pregabalina, $\alpha-2$ agonistas ou cetamina em cirurgias de membro superior $^{17}$.

Adicionalmente, pacientes que receberam petidina ou cetoprofeno como adjuvantes da lidocaína em anestesia regional intravenosa, apresentaram zumbido, tontura, náusea, vômito e prurido. Nesse estudo, nenhum paciente apresentou depressão respiratória significativa e os efeitos secundários não foram estatisticamente significativos $^{11}$.

Esta revisão classificou $18,75 \%$ dos artigos como inseguros. Tal número se relaciona com complicaçôes gastrointestinais que incidiram em alguns pacientes, causando risco de vida devido às perfurações e hemorragias. No entanto, essas complicaçóes ocorreram mais frequentemente em pacientes susceptíveis que apresentavam os seguintes fatores de risco: uso prolongado de AINEs para o tratamento de artrite reumatoide ou osteoartrite, idade superior a 65 anos, comorbidades, dose elevada, complicaçóes gastrointestinais anteriores e uso de aspirina. Por outro lado, não foi observado, em nossa revisão, um aumento significante do uso de inibidores seletivos da COX-2, com o objetivo de reduzir esses efeitos adversos gastrointestinais. Provavelmente, esse resultado reflete um certo temor em função da propriedade dessa subclasse de AINEs em aumentar o risco de eventos trombóticos, ainda que esse efeito ocorra mais frequentemente com o seu uso prolongado ${ }^{18,19}$.

Do mesmo modo, o uso prolongado de AINEs, após o primeiro infarto do miocárdio, aumenta o risco de óbito em até 5 anos após o evento ${ }^{20}$. Isso mostra que estes medicamentos não têm janela terapêutica aparentemente segura para tais pacientes. Assim, nota-se que a segurança também está relacionada à indicação adequada.

Outro aspecto abordado foi a "segurança não estabelecida" que incluiu 6,25\% dos artigos. Esse aspecto está relacionado com o risco de aumento de baixo peso ao nascer associado ao diclofenaco e ao ibuprofeno, o qual foi atribuído a fatores ainda não totalmente elucidados e, portanto, necessitando de novos estudos ${ }^{19}$.

\section{CONCLUSÃO}

Portanto, os artigos analisados nesse estudo mostraram que os AINEs são eficazes no controle da dor aguda de intensidade leve a moderada e têm um perfil aceitável de segurança. $\mathrm{O}$ efeito analgésico foi observado com a utilização isolada do AINE ou em associação com outros fármacos quando era empregada a estratégia de tratamento multimodal. Por outro lado, estudos adicionais, com amostras maiores, são fundamentais para corroborar os resultados encontrados nesta revisão.

\section{REFERÊNCIAS}

1.Batlouni M. Anti-inflamatórios nấo esteroides: Efeitos cardiovasculares, cerebrovasculares e renais. Arq Bras Cardiol 2010;94:556-63. http://dx.doi. org/10.1590/S0066-782X2010000400019

2.Ridderikhof ML, Lirk P, Schep NW, Hoeberichts A, Goddijn WT, Luitse JS, et al. The PanAM study: a multi-center, double-blinded, randomized, non-inferiority study of paracetamol versus non-steroidal anti-inflammatory drugs in treating acute musculoskeletal trauma. BMC Emerg Med 2013;13:19. http:// dx.doi.org/10.1186/1471-227X-13-19

3.Laine L, Kivitz AJ, Bello AE, Grahn AY, Schiff MH, Taha AS. Double-blind randomized trials of single-tablet ibuprofen/high-dose famotidine vs. Ibuprofen alone for reduction of gastric and duodenal ulcers. Am J Gastroenterol 2012;107:379-86. http://dx.doi.org/10.1038/ajg.2011.443

4.Landgraf CS, Marques RC, Pires OC, Constantino E, Leite VR, Posso MBS, et al. Avaliação da analgesia pós-operatória em um hospital universitário. Rev Dor 2010;11:319-22.

5.Martinez JE, Grassi DC, Marques LG. Análise da aplicabilidade de três instrumentos de avaliaçấo de dor em distintas unidades de atendimento: ambulatório, enfermaria e urgência. Rev Bras Reumatol 2011;51:299-308. http:// dx.doi.org/10.1590/S0482-50042011000400002

6.Pereira RJ, Munechika M, Sakata RK. Pain management after outpatient surgical procedure. Rev Dor 2013;14: 61-7. http://dx.doi.org/10.1590/ $\underline{\text { S1806-00132013000100015 }}$

7.Mathiesen O, Dahl B, Thomsen BA, Kitter B, Sonne N, Dahl JB, et al. A comprehensive multimodal pain treatment reduces opioid consumption after multilevel spine surgery. Eur Spine J 2013;22:2089-96. http://dx.doi. org/10.1007/s00586-013-2826-1

8.Scheiman JM, Hindley CE. Strategies to optmise treatment with NSAIDs in patients at risk for gastrointestinal and cardiovascular adverse events. Clin Ther 2010;32:667-77. http://dx.doi.org/10.1016/j.clinthera.2010.04.009

9.Farshchi A, Ghiasi G. Comparison the analgesic effects of single dose administration of tramadol or piroxicam on postoperative pain after cesarean delivery. Acta Med Iran 2010;48:148-53.

10.Bidese BL, Sakuma KA, Júnior AA, Sartor MC. Postoperative analgesia by non-specialists in pain. Rev Dor 2014;15:36-40. http://dx.doi. org/10.5935/1806-0013.20140009

11.Desai SN, Santhosh MCB. Estudo comparativo, randômico e duplo-cego 
de petidina e cetoprofeno como adjuvantes de lidocaína em anestesia regional intravenosa. Rev Bras Anestesiol 2014;64:221-6. http://dx.doi.org/10.1016/j. bjan.2013.03.016

12.Barbosa MH, Araújo NF, Silva JAJ, Corrêa TB, Moreira TM, Andrade EV. Avaliaçáo da intensidade da dor e analgesia em pacientes no período pós-operatório de cirurgias ortopédicas. Esc Anna Nery 2014;18:143-7. http://dx.doi. org/10.5935/1414-8145.20140021

13.Sertoz N, Kocaoglu N, Ayanoğlu HO. Comparação de lornoxicam e fentanil adicionados à lidocaína em anestesia regional intravenosa. Rev Bras Anestesiol 2013;63:311-6. http://dx.doi.org/10.1016/j.bjan.2013.06.001

14.Kara C, Resorlu B, Cicekbilek I, Unsal A. Analgesic efficacy and safety of nonsteroidal anti-inflammatory drugs after transurethral resection of prostate. Int Braz J Urol 2010;36:49-54. http://dx.doi.org/10.1590/S167755382010000100008

15.Husted H, Lunn TH, Troelsen A, Gaarn-Larsen L, Kristensen BB, Kehlet H. Why still in hospital after fast-track hip and knee arthroplasty? Acta Orthop 2011;82:679-84. http://dx.doi.org/10.3109/17453674.2011.636682

16.Defrance C, Bousquet PJ, Demoly P. Evaluating the negative predictive value of provocation tests with nonsteroidal anti-inflammatory drugs. Allergy 2011;66:1410-4. http://dx.doi.org/10.1111/j.1398-9995.2011.02671.x

17.Lee SH, Lee JW, Choy WS. Is multimodal analgesia as effective as postoperative patient-controlled analgesia following upper extremity surgery? Orthop Traumatol Surg Res 2013;99:895-901. http://dx.doi.org/10.1016/j. otsr.2013.09.005

18.Lee SH, Han CD, Yang IH, Ha CW. Prescription pattern of NSAIDs and the prevalence of NSAID-induced gastrointestinal risk factors of orthopaedic patients in clinical practice in Korea. J Korean Med Sci 2011;26:561-7. http:// dx.doi.org/10.3346/jkms.2011.26.4.561

19.Nezvalová-Henriksen K, Spigset O, Nordeng H. Effects of ibuprofen, di- clofenac, naproxen, and piroxicam on the course of pregnancy and pregnancy outcome: a prospective cohort study. BJOG 2013;120:948-59. http://dx.doi. org/10.1111/1471-0528.12192

20.Oslen MAS, Fosbøl EL, Lindhardsen J, Folke F, Charlot M, Selmer C, et al. Long-term cardiovascular risk of nonsteroidal anti-Inflammatory drug use according to time passed after first-time myocardial infarction. Circulation 2012;126:1955-63. http://dx.doi.org/10.1161/CIRCULATIONAHA.112.112607

21.Andersen LØ, Otte KS, Husted H, Gaarn-Larsen L, Kristensen B, Kehlet H. High-volume infiltration analgesia in bilateral hip arthroplasty: a randomized, double-blind placebo-controlled trial. Acta Orthop 2011;82:423-6. http://dx.doi.org/10.3109/17453674.2011.596063

22.Mezentsev VA. Meta-analysis of the efficacy of non-steroidal anti-inflammatory drugs vs. opioids for SWL using modern electromagnetic lithotripters. Int Braz J Urol 2009;35:293-8.

23.Chan SSM, Luben R, Bergmann MM, Boeing H, Olsen A, Tjonneland A, et al. Aspirin in the aetiology of Crohn's disease and ulcerative colitis: a European prospective cohort study. Aliment Pharmacol Ther 2011;34:649-55. http:// dx.doi.org/10.1111/j.1365-2036.2011.04784.x

24.Vega CG, Speed C, Wolfarth B, González J. Traumeel vs. diclofenac for reducing pain and improving ankle mobility after acute ankle sprain: A multicentre, randomised, blinded, controlled and non-inferiority trial. Int J Clin Pract 2013;67:979-89. http://dx.doi.org/10.1111/ijcp.12219

25.Boonriong T, Tangtrakulwanich B, Glabglay P, Nimmaanrat S. Comparing etoricoxib and celecoxib for preemptive analgesia for acute postoperative pain in patients undergoing arthroscopic anterior cruciate ligament reconstruction: a randomized controlled trial. BMC Musculoskelet Disord 2010;11:246. http://dx.doi.org/10.1186/1471-2474-11-246 